\title{
Les monoxyde d'azote (NO) synthases
}

NO - médiateur gazeux aux multiples fonctions $\left(\mathrm{m} / \mathrm{s} n^{\circ} 10\right.$, vol. 7, p. 1094, Tableau I) - est un radical libre, produit de la catalyse enzymatique de la L-arginine en citrulline. Cette catalyse est assurée de façon spécifique de tissu par une famille d'enzymes - les monoxyde d'azote synthases - dont trois représentantes ont maintenant leur ADN complémentaire cloné (Tableau I). Ces isoformes se partagent en deux groupes: les NO synthases constitutives et les NO synthases inductibles.

Membres du premier groupe, les NO synthases endothéliale et neuronale, initialement considérées comme produits d'un même gène, sont en fait codées par deux gènes différents $[1,2]$. En effet, l'isoenzyme endothéliale bovine - produit d'expression dans des cellules COS d'un ADN complémentaire isolé après criblage d'une banque issue de cellules endothéliales - ne présente que $60 \%$ d'homologie au niveau de la séquence peptidique avec l'isoenzyme neuronale de la même espèce, et l'existence d'au moins deux gènes codant respectivement pour chacune de ces enzymes est confirmée par le profil de migration de fragments de restriction obtenus après hybridation avec des sondes spécifiques (Southern blot) [2]. Toutes deux ont en commun des domaines de reconnaissance du complexe $\mathrm{Ca}^{2+} /$ calmoduline et des transporteurs de protons FAD, FMN et NADPH. L'isoforme neuronale, de masse moléculaire plus importante, possède un domaine $\mathrm{NH} 2$ terminal supplémentaire d'environ 200 acides aminés, dont la fonction est à ce jour inconnue. Ces deux enzymes ont une activité basale stimulable après activation : (1) des récepteurs membranaires type NMDA par les acides aminés neuroexcitateurs pour la forme neuronale, ou (2) des récepteurs endothéliaux reconnaissant divers vasomédiateurs relaxants, dont l'acétylcholine et les bradykinines, pour la forme endothéliale.

A l'inverse des isoformes neuronale et endothéliale, l'isoforme macrophagique, présente aussi dans le polynucléaire neutrophile, indépendante du complexe $\mathrm{Ca}^{2+} /$ calmoduline, est inductible : l'apparition d'une activité NO synthase, après stimulation de lignées cellulaires macrophagiques par les LPS bactériens, l'interféron $\gamma$ ou l'IL-1, est prévenue par un inhibiteur de la synthèse de protéines $[3,4]$. La toxine staphylococcique TSST-1 (toxic shock sndrome toxin 1) stimule aussi la production macrophagique de $\mathrm{NO}$ ex vivo, suggérant l'implication de ce dernier dans les manifestations hémodynamiques du toxic shock syndrome staphylococcique [5]. Une autre isoforme hépatique et inductible par des endotoxines a récemment été décrite [6]. Non encore clonée, elle possède la propriété curieuse d'avoir son activité fortement dépendante de la présence de calmoduline, mais largement indépendante de la concentration en calcium. Cette particularité pourrait avoir des conséquences importantes sur les plans pharmacologique et thérapeutique, dans la mesure où de nombreux chocs septiques à bacilles Gram négatif sont à point de départ digestif, et s'accompagnent d'un passage hépatique obligatoire d'une quantité importante d'endotoxines.

En effet, l'emploi d'inhibiteurs de la synthèse du NO - comme la $\mathrm{N}^{\mathrm{G}}$ monométhyl L-arginine (L-NMMA) ou la $\mathrm{N}^{\mathrm{G}}$ nitro L-arginine méthyl ester (L-NAME) - dans le traitement des manifestations hémodynamiques du choc septique ou de l'insuffisance hépatique a été récemment rapporté chez l'animal et chez l'homme, avec des

\begin{tabular}{|c|c|c|c|}
\hline \multicolumn{4}{|c|}{$\begin{array}{c}\text { Tableau I } \\
\text { NO SYNTHASES : LES DIFFÉRENTES ISOFORMES }\end{array}$} \\
\hline Spécificité & $\begin{array}{c}\text { Cellule } \\
\text { endothéliale }\end{array}$ & Neurone & Macrophage \\
\hline Masse moléculaire & 135 kDa & $150 \mathrm{kDa}$ & $130 \mathrm{kDa}$ \\
\hline Activité & $\begin{array}{l}\text { Constitutive } \\
\text { et stimulable }\end{array}$ & $\begin{array}{l}\text { Constitutive } \\
\text { et stimulable }\end{array}$ & Inductible \\
\hline $\begin{array}{c}\text { Fonction } \\
\text { du produit NO }\end{array}$ & $\begin{array}{l}\text { EDRF } \\
\text { (endothelium- } \\
\text { derived relaxing } \\
\text { factor) }\end{array}$ & Neurotransmetteur & $\begin{array}{l}\text { Bactéricidie } \\
\text { Tumoricidie }\end{array}$ \\
\hline $\begin{array}{c}\text { Cofacteur } \mathrm{Ca}^{2+} / \\
\text { Calmoduline }\end{array}$ & Dépendante & Dépendante & Indépendante \\
\hline $\begin{array}{c}\text { Cofacteurs FAD, } \\
\text { FMN, NADPH }\end{array}$ & Dépendante & Dépendante & Dépendante \\
\hline $\begin{array}{c}\text { Effecteurs } \\
\text { membranaires }\end{array}$ & $\begin{array}{l}\text { Acétylcholine } \\
\text { Bradykinine } \\
\text { Sérotonine - ATP }\end{array}$ & $\begin{array}{l}\text { Acides aminés } \\
\text { neuro-excitateurs }\end{array}$ & $\begin{array}{l}\text { Lipopoly- } \\
\text { saccharides } \\
\text { Interféron } \\
\text { Interleukine } 1\end{array}$ \\
\hline $\begin{array}{l}\text { Effet inhibiteur } \\
\text { de la } \\
\mathrm{N} \text {-monométhyl- } \\
\text { arginine }\end{array}$ & Présent & Présent & Présent \\
\hline
\end{tabular}


résultats variables et controversés ([7-10]; $m / s n^{\circ} 2$, vol. 8, p. 187). La principale critique faite à un tel traitement est que, bien qu'elle contribue à une remontée de la pression artérielle systémique, la suppression de la synthèse endothéliale de $\mathrm{NO}$ majore localement l'hypoperfusion et l'ischémie de nombreux tissus, notamment le myocarde, et pourrait favoriser la survenue de poussées d'hypertension artérielle pulmonaire en cas de syndrome de détresse respiratoire de l'adulte (SDRA), complication fréquente des septicémies à bacilles Gram négatif et des chocs septiques. L'absence de spécificité de ces analogues a aussi pour effet d'inhiber la NO synthase macrophagique, privant ainsi l'organisme du pouvoir bactéricide de NO. Enfin, chez l'animal, l'effet de ces inhibiteurs est dépendant de la dose, avec des conséquences hémodynamiques variables selon la posologie [8]. Le clonage des ADN complémentaires codant pour les isoformes non macrophagiques et inductibles par les endotoxines, et leur expression dans un système cellulaire aspécifique, pourraient donc constituer l'étape préalable à la caractérisation d'inhibiteurs spécifiques utilisables lors de situations septiques graves, devenues l'une des principales causes de mortalité dans les unités de réanimation.

Au niveau endothélial, NO a un effet paracrine vaso-relaxant sur la cellule musculaire lisse, via la production de GMP cyclique et l'activation de protéine kinases dépendantes du GMP cyclique. Son instabilité intrinsèque et sa forte affinité pour les groupements prosthétiques héminiques, dont bien sûr celui de l'hémoglobine, rendent compte d'une durée de vie de l'ordre de quelques secondes. Cette caractéristique est l'une des raisons pour lesquelles NO a très rapidement acquis, en pathologie cardio-respiratoire, un potentiel thérapeutique considérable [11]. Il trouve aujourd'hui la principale de ses indications dans certaines formes d'hypertension artérielle pulmonaire, notamment celles secondaires à une hypoxie alvéolaire [12, 13]. Chez l'adulte, l'inhalation d'un mélange gazeux contenant entre 10 et $40 \mathrm{ppm}$ de NO produit une baisse significative et durable des résistances pulmonaires de patients atteints d'hypertension arté- syndrome de détresse respiratoire aigu de l'adulte [14, 15]. L'avantage de cette voie d'administration est la spécificité d'action sur la circulation pulmonaire, l'inactivation quasi immédiate du gaz permise par la fixation à l'hémoglobine s'effectuant avant le retour veineux pulmonaire. La persistance d'une hypertension artérielle pulmonaire à la naissance, grave syndrome fréquemment observé chez le nouveau-né [16], pourrait aussi constituer une autre indication de l'inhalation de mélanges gazeux enrichis en NO. NO semble en effet jouer un rôle important dans la baisse des résistances vasculaires pulmonaires qui caractérise l'adaptation cardio-respiratoire du mammifère nouveau-né à la vie extrautérine. Ajoutons enfin que des données expérimentales et préliminaires suggèrent que ce même mode d'administration pourrait trouver des indications dans les syndromes bronchiques obstructifs aigus ou chroniques [17]. Dans toutes ces situations, le danger d'un tel traitement est moins l'apparition d'une faible méthémoglobinémie que la production - favorisée par une fraction d'O $\mathrm{O}_{2}$ inspirée élevée - de $\mathrm{NO}_{2}$, extrêmement toxique et potentiellement mutagène pour l'épithélium respiratoire [17]. Son usage présuppose donc une surveillance étroite des concentrations de $\mathrm{NO}$ inspiré et de $\mathrm{NO}_{2}$ expiré [18], et une évaluation approfondie de sa toxicité pulmonaire chez l'animal.

NO est aussi un neurotransmetteur, non seulement du système nerveux central, mais aussi du système nerveux périphérique. C'est ainsi le premier neurotransmetteur non cholinergique, non adrénergique, et médiateur de la relaxation du tube digestif, caractérisé à ce jour [20]. Il participe au contrôle neuronal du tonus vasculaire cérébral [21], de l'érection [22], et de la sécrétion d'insuline par les cellules $\beta$ des îlots de Langerhans [23]. Dans le cerveau, avec l'exception du cervelet - très riche en NO synthase neuronale - la production de NO est limitée à une petite fraction de la population neuronale du cortex, de l'hippocampe et du corpus striatum. Ces mêmes populations neuronales sont caractérisées par une activité NADPH diaphorase, et par leur résistance à l'hypoxie in vivo ou à la toxicité de l'acide glutamique (acide aminé neuro- excitateur) ex vivo. Co-cultivés en présence de neurones producteurs de NO stimulés par l'acide glutamique, les neurones non producteurs de $\mathrm{NO}$ meurent, sauf si le milieu est conditionné par l'absence d'arginine ou additionné de NMMA. Tous ces faits sont en faveur d'un rôle important joué par NO dans la physiopathologie de l'ischémie cérébrale, de certaines affections neurodégénératives, et peutêtre, sécrété alors par les monocytes et macrophages cérébraux, dans l'encéphalite du SIDA [24]. L'importance du monoxyde d'azote en neurophysiopathologie apparaît donc considérable et pose le problème de la nature exacte des fonctions neurophysiologiques de NO, comme celui - ce n'est pas une plaisanterie - de l'existence d'autres neurotransmetteurs gazeux comme... le monoxyde de carbone CO [24, 25]

Thierry Lacaze-Masmonteil Inserm U. 129, 24, nue du Faubourg Saint-Jacques, 75014 Paris, France.

\section{RÉFÉRENCES}

1. Bredt DS, Hwang PM, Glatt $\mathrm{CH}$, Lowenstein C, Reed RR, Snyder SH. Cloned and expressed nitric oxide synthase structurally ressembles cytochrome p-450 reductase. Nature 1991; 351 : 714-8.

2. Lamas S, Marsden P, Li GK, Tempst P, Michel T. Endothelial nitric oxide synthase : molecular cloning and characterization of a distinct constitutive enzyme isoform. Proc Natl Acad Sci USA 1992 ; 89 : 6348-52.

3. Lyons CR, Orloff GJ, Cunningham JM. Molecular cloning and functional expression of an inducible nitric oxide synthase from a murine macrophage cell line. J Biol Chem $1992 ; 267: 6370-4$.

4. Lowenstein CJ, Glatt CS, Bredt DS, Snyder SH. Cloned and expressed macrophage nitric oxide synthase contrasts with the brain enzyme. Proc Natl Acad Sci USA 1992 ; $89: 6711-5$.

5. Zembowicz A, Vane JR. Induction of nitric oxide synthase activity by toxic shock syndrome toxin 1 in a macrophage-monocyte cell line. Proc Natl Acad Sci USA 1992 ; 89 : 2051-5.

6. Evans T, Carpenter A, Cohen J. Purification of a distinctive form of endotoxininduced nitric oxide synthase from rat liver. Proc Natl Acad Sci USA 1992 ; 89 : 5361-5. 7. Petros A, Bennett D, Vallance P. Effect of nitric oxide synthase inhibitors on hypotension in patients with septic shock. Larcet 1991 ; 338 : 1557-8.

8. Nava E, Palmer RM, Moncada S. Inhibition of nitric oxide synthesis in septic shock : how much is beneficial ? Lancet 1991; 338 : 1555-7. 
9. Hotchkiss RS, Karl IE, Parker JL, Adams HR. Inhibition of NO synthesis in septic shock. Lancet 1992 ; 339 : 434-5.

10. Cohen J, Silva A. NO inhibitors and septic shock. Lancet $1992 ; 339$ : 751.

11. Collier J, Vallance P. Physiological importance of nitric oxide: an endogenous nitrovasodilator. $\mathrm{Br}$ Med J 1991 ; 302: 1289-90.

12. Frostell C, Fratacci MD, Wain JC, Jones $\mathrm{R}$, Zapol WM. Inhaled nitric oxide, a selective pulmonary vasodilatator reversing hypoxic pulmonary vasoconstriction. Circulation 1991 ; 83: 2038-47.

13. Adnot S, Raffestin B, Eddahibi S, Braquet $\mathrm{P}$, Chabrier PE. Loss of endotheliumdependant relaxant activity in the pulmonary circulation of rats exposed to chronic hypoxia. J Clin Invest 1991 ; 87 : 155-62.

14. Pepke-Zaba J, Higenbottam TW, Tuan Dinh-Xuan A, Stone D, Wallwork J. Inhaled nitric oxide as a cause of selective pulmonary vasodilatation in pulmonary hypertension. Lancet 1991 ; 338 : 1173-4.

15. Falke K, Rossaint R, Pison V, et al. Inhaled nitric oxide selectively reduces pulmonary hypertension in severe ARDS and improves gas exchanges as well as right heart ejection fraction : a case report. Am Rev Resp Dis $1991 ; 143$ : A248.

16. Lacaze-Masmonteil T, Zupan V, Dehan M. Hypertension artérielle pulmonaire persistante du nouveau-né : progrès et espoirs. Arch Fr Pediatr 1991 ; 48 : 597-601.

17. Dupuy PM, Shore SA, Drazen JM, Fzostell C, Hill WA, Zapol WM. Bronchodilator action of inhaled nitric oxide in guinea pigs. J Clin Invest 1992 ; 92 : 421-8.

18. Nguyen $T$, Brunson $D$, Crespi CL, Penman BW, Wishnok JS, Tannenbaum SR. DNA damage and mutation in human cells exposed to nitric oxide in vitro. Proc Natl Acad Sci USA 1992; 89 : 3030-4.

19. Foubert L, Fleming B, Latimer R, Jonas $M$, Oduro A, Borland C, Higenbottam T. Safety guidelines for use of nitric oxide. Lancet 1992 ; 339 : 1615-6.

20. Desai KM, Sessa WC, Vane JR. Involvement of nitric oxide in the reflex relaxation of the stomach to accommodate food and fluid. Nature 1991 ; 351 : 477-9.

21. Beckman JS. The double-edged role of nitric oxide in brain function and superoxidemediated injury. J Dev Physiol 1991; 15 : 53-9.

22. Rajfer J Aronson WJ, Bush PA, Dorey FJ, Ignarro LJ. Nitric oxide as a mediator of relaxation of the corpus cavernosum in response to non-adrenergic, non-cholinergic neurotransmission. N Engl J Med 1992; 326 : 90-4.

23. Schmidt HHHW, Warner TD, Ishii K, Sheng $H$, Murad $F$. Insulin-secretion from pancreatic B-cells caused by L-arginine-derived nitrogen oxides. Science 1992; 255 : 721-3.

24. Snyder SH. Nitric oxide : first in a new class of neurotransmitters? Science $1992 ; 257$ : 494-6.

25. Lowenstein CJ, Snyder SH. Nitric oxide, a novel biologic messenger. Cell $1992 ; 70$ : 705-7.

\section{TIRÉS A PART}

Th. Lacaze-Masmonteil.

$m / s n^{\circ} 8$, vol. 8, octobre 92 